Electron spin dynamics in quantum dots and related nanostructures due to hyperfine interaction with nuclei

This article has been downloaded from IOPscience. Please scroll down to see the full text article.

2003 J. Phys.: Condens. Matter 15 R1809

(http://iopscience.iop.org/0953-8984/15/50/R01)

View the table of contents for this issue, or go to the journal homepage for more

Download details:

IP Address: 132.199.98.211

The article was downloaded on 23/05/2013 at 09:51

Please note that terms and conditions apply. 
TOPICAL REVIEW

\title{
Electron spin dynamics in quantum dots and related nanostructures due to hyperfine interaction with nuclei
}

\author{
John Schliemann, Alexander Khaetskii and Daniel Loss \\ Department of Physics and Astronomy, University of Basel, CH-4056 Basel, Switzerland
}

Received 1 September 2003

Published 3 December 2003

Online at stacks.iop.org/JPhysCM/15/R1809

\begin{abstract}
We review and summarize recent theoretical and experimental work on electron spin dynamics in quantum dots and related nanostructures due to hyperfine interaction with surrounding nuclear spins. This topic is of particular interest with respect to several proposals for quantum information processing in solid state systems. Specifically, we investigate the hyperfine interaction of an electron spin confined in a quantum dot in an s-type conduction band with the nuclear spins in the dot. This interaction is proportional to the square modulus of the electron wavefunction at the location of each nucleus leading to an inhomogeneous coupling, i.e. nuclei in different locations are coupled with different strengths. In the case of an initially fully polarized nuclear spin system an exact analytical solution for the spin dynamics can be found. For not completely polarized nuclei, approximation-free results can only be obtained numerically in sufficiently small systems. We compare these exact results with findings from several approximation strategies.
\end{abstract}

\section{Contents}

1. Introduction

2. Electron spin dynamics in quantum dots

3. Hyperfine interaction in semiconductors

4. Electron spin dynamics in quantum dots due to hyperfine interaction with nuclei

4.1. Modelling hyperfine interaction in quantum dots

4.2. Different types of initial states

4.3. Numerical results for electron spin dynamics: product states versus randomly correlated states

4.4. Decoherence and the generation of entanglement

4.5. Averaging over nuclear configurations.

Dephasing time for an ensemble of dots

4.6. Further approximate studies of electron spin dynamics

4.7. Further studies and developments 
5. Conclusions and outlook 1831

Acknowledgments 1831

$\begin{array}{ll}\text { References } & 1831\end{array}$

\section{Introduction}

In recent years an extraordinary and increasing interest in spin-dependent phenomena in semiconductors has developed in the solid state physics community [1,2]. These research activities are often labelled by the keyword 'spintronics' which summarizes the entire multitude of efforts towards using the electron spin rather than, or in combination with, its charge, for information processing, or even more ambitiously, quantum information processing. In fact, in recent years a series of proposals for implementing quantum computation in solid state systems using electron and/or nuclear spins have been put forward [2-8]. In order to use the electron spin as an information carrier, long spin decoherence times are desirable if not indispensable. A serious possible limitation of spin coherence in semiconductors is the hyperfine interaction with surrounding nuclear spins. In fact, in semiconductors isotopes carrying a nonzero magnetic moment are ubiquitous. The commercial use of semiconductor technology is, so far, grossly dominated by silicon applications. In this material the magnetic isotope ${ }^{29} \mathrm{Si}$, having a spin $1 / 2$ and a magnetic moment of -0.5553 nuclear magnetons, occurs with a natural abundance of $4.7 \%$, together with the two spinless stable isotopes ${ }^{28} \mathrm{Si}$ and ${ }^{30} \mathrm{Si}$. From these numbers, hyperfine interaction might not appear to be a particularly relevant issue. However, the systems presently mostly studied in the field of spin electronics and solid state quantum information processing contain materials such as $\mathrm{GaAs}$, $(\mathrm{Ga}, \mathrm{Al}) \mathrm{As}$ and InAs whose elements consist entirely of spin-carrying isotopes with substantial magnetic moments. As will be discussed in detail in the present review, in such systems the hyperfine coupling of electron spins to nuclear spins can easily become an important interaction. In table 1 we summarize the natural abundances and magnetic properties of stable spin-carrying nuclei relevant to semiconductor systems.

A principal way of avoiding such hyperfine couplings to electron spins is to use isotopically purified material containing only a greatly reduced amount of magnetic isotopes [9]. However, present technology allows isotope purification of typical semiconductor materials only up to a few hundredths of a per cent or more of unwanted isotopes remaining. This degree of purification might, in general, not be sufficient to meet the high precision demands of implementations of quantum information processing. Moreover, such isotopically purified materials have often quite high prices such that they might not appear as a technologically viable option even if the demands on precision were lower.

This review is organized as follows: in section 2 we summarize important experimental work on electron spin dynamics in semiconductor nanostructures which have motivated the mostly theoretical studies we are reviewing in this paper. In the following section we describe the basic physics of the hyperfine interaction between electron and nuclear spins as it occurs in semiconductors. Section 4 is devoted to a detailed analysis of electron spin dynamics in quantum dots due to hyperfine interaction with nuclear spins. After specifying the details of the model in section 4.1, we discuss the ground state and elementary properties of the underlying Hamiltonian in section 4.1.1. There we also describe a full analytical solution for the electron spin dynamics which can be obtained in the case of an initially fully polarized nuclear spin system. In section 4.1.2 we outline how to solve for the eigenstates and energy levels of the system via the Bethe ansatz technique, relying on the integrability of the hyperfine coupling Hamiltonian. In section 4.2 we introduce different types of initial states for the nuclear spin system. These different types of initial states lead to significantly different electron spin 
Table 1. Natural abundances, total nuclear spin quantum numbers $I$ and nuclear magnetic moments $\mu_{I}$ of spin-carrying stable nuclei relevant to semiconductor materials. The values for $\mu_{I}$ are given in units of the nuclear magneton $\mu_{\mathrm{N}}=e \hbar / 2 m_{\mathrm{p}}$, where $m_{\mathrm{p}}$ is the proton mass. The data are adopted from [71].

\begin{tabular}{|c|c|c|c|}
\hline & $\begin{array}{l}\text { Natural } \\
\text { abundance }(\%)\end{array}$ & $I$ & $\mu_{I}$ \\
\hline${ }^{9} \mathrm{Be}$ & 100 & $3 / 2$ & -1.1776 \\
\hline${ }^{10} \mathrm{~B}$ & 19.78 & 3 & +1.8007 \\
\hline${ }^{11} \mathrm{~B}$ & 80.22 & $3 / 2$ & +2.6885 \\
\hline${ }^{13} \mathrm{C}$ & 1.11 & $1 / 2$ & +0.7024 \\
\hline${ }^{14} \mathrm{~N}$ & 99.63 & 1 & +0.4036 \\
\hline${ }^{15} \mathrm{~N}$ & 0.37 & $1 / 2$ & -0.2831 \\
\hline${ }^{27} \mathrm{Al}$ & 100 & $5 / 2$ & +3.6414 \\
\hline${ }^{29} \mathrm{Si}$ & 4.70 & $1 / 2$ & -0.5553 \\
\hline${ }^{31} \mathrm{P}$ & 100 & $1 / 2$ & +1.1317 \\
\hline${ }^{33} \mathrm{~S}$ & 0.76 & $3 / 2$ & +0.6433 \\
\hline${ }^{67} \mathrm{Zn}$ & 4.11 & $5 / 2$ & +0.8754 \\
\hline${ }^{69} \mathrm{Ga}$ & 60.4 & $3 / 2$ & +2.016 \\
\hline${ }^{71} \mathrm{Ga}$ & 39.6 & $3 / 2$ & +2.562 \\
\hline${ }^{73} \mathrm{Ge}$ & 7.76 & $9 / 2$ & -0.8792 \\
\hline${ }^{75} \mathrm{As}$ & 100 & $3 / 2$ & +1.439 \\
\hline${ }^{77} \mathrm{Se}$ & 7.58 & $1 / 2$ & +0.534 \\
\hline${ }^{111} \mathrm{Cd}$ & 12.75 & $1 / 2$ & -0.5943 \\
\hline${ }^{113} \mathrm{Cd}$ & 12.26 & $1 / 2$ & -0.6217 \\
\hline${ }^{113} \mathrm{In}$ & 4.28 & $9 / 2$ & +5.523 \\
\hline${ }^{115} \mathrm{In}$ & 95.72 & $9 / 2$ & +5.534 \\
\hline${ }^{115} \mathrm{Sn}$ & 0.35 & $1 / 2$ & -0.918 \\
\hline${ }^{117} \mathrm{Sn}$ & 7.16 & $1 / 2$ & -1.000 \\
\hline${ }^{119} \mathrm{Sn}$ & 8.58 & $1 / 2$ & -1.046 \\
\hline${ }^{121} \mathrm{Sb}$ & 57.25 & $5 / 2$ & +3.359 \\
\hline${ }^{123} \mathrm{Sb}$ & 42.75 & $7 / 2$ & +2.547 \\
\hline${ }^{123} \mathrm{Te}$ & 0.87 & $1 / 2$ & -0.7357 \\
\hline${ }^{125} \mathrm{Te}$ & 6.99 & $1 / 2$ & -0.8871 \\
\hline${ }^{199} \mathrm{Hg}$ & 16.84 & $1 / 2$ & +0.5027 \\
\hline${ }^{201} \mathrm{Hg}$ & 13.22 & $3 / 2$ & -0.5567 \\
\hline${ }^{207} \mathrm{~Pb}$ & 22.6 & $1 / 2$ & +0.5895 \\
\hline${ }^{209} \mathrm{Bi}$ & 100 & $9 / 2$ & +4.080 \\
\hline
\end{tabular}

dynamics, which are described in section 4.3. The results reported on there are based on exact diagonalizations of the Hamiltonian for sufficiently small systems. In section 4.4 we discuss the intimate connection between the decay of the electron spin and the generation of quantum entanglement between the electron spin and the nuclear spin system. Section 4.5 is devoted to the important question of spin dephasing in an ensemble of dots, as opposed to decoherence of a single electron spin. In section 4.6 we discuss several further approximate treatments of the electron spin dynamics that have appeared in the recent literature and compare them with the (quasi-)approximation-free approaches described before. Further, mostly theoretical, work relevant to the issue of hyperfine interaction between an electron spin bound to a quantum dot and surrounding nuclear spins is summarized in section 4.7. We close with conclusions and an outlook to the future in section 5 . 


\section{Electron spin dynamics in quantum dots}

The dynamics of electron spins confined in semiconductor nanostructures is a rich and very active field. Among the most notable developments are experiments by Kikkawa and Awschalom $[10,11]$ who demonstrated remarkably large coherence times for electron spins in n-doped bulk GaAs. This timescale can exceed $100 \mathrm{~ns}$ and represents the $T_{2}^{*}$ time, i.e. the transverse spin relaxation time of an ensemble of electrons. These experimental findings have generated a great deal of prospects in the fields of spin electronics and semiconductor quantum computing $[1,2]$.

A photoluminescence study of excitons localized in single GaAs quantum dots was done by Gammon et al [12]. In a subsequent study of the magnetooptical spectra of individual localized excitons [13] the role of electron spin hyperfine interaction with nuclear spins was investigated. Though these experiments dealt with single GaAs quantum dots (not ensembles of them), it was not the electron spin dynamics studied but spectra of individual localized excitons, where the strong Coulomb interaction between an electron and a heavy hole is important. The relaxation lifetime of electron spins for an ensemble of CdSe quantum dots of very small diameter (20-80 ̊) was measured by Gupta et al [14] using a femtosecond-resolved Faraday rotation technique. Again, due to the small size of the dot the Coulomb interaction between electron and hole is not negligible. Therefore, it is highly probable that the observed short relaxation time (being of the order of nanoseconds) is due to fast spin dynamics of the hole. The surface states could also contribute to the spin relaxation due to the small size of the dot. Finally Epstein et al [15] have analysed the spin lifetime of photogenerated carriers in InAs quantum dots using the Hanle effect.

The above experiments $[14,15]$ were performed on ensembles of quantum dots, not single dots. Therefore the estimated spin relaxation times are $T_{2}^{*}$ timescales. The experiments which directly probe the single-electron spin relaxation were done recently by Fujisawa et al [16] and Hanson et al [17]. The non-equilibrium tunnelling current through excited states in an $\mathrm{AlGaAs} / \mathrm{GaAs}$ quantum dot was studied using a pulse-excitation technique which measures the energy relaxation time from the excited state to the ground state. Very low spin-flip rates were observed, which are consistent with the theoretical predictions [18-20]. The physical mechanisms for electron spin relaxation for delocalized states include the interplay of spin-orbit coupling with impurity scattering and/or electron-phonon interaction and the hyperfine interaction with surrounding nuclear spins. In recent theoretical studies Khaetskii and Nazarov $[19,20]$ have concluded that the first type of mechanism is strongly suppressed for electrons localized in quantum dots, see also [21]. As for the contribution to electron spin decoherence due to the combined effect of the spin-orbit interaction and the spinindependent interaction with acoustic phonons, there is an indication that, within the Markovian approximation, which is usually applicable to this problem, $T_{2}$ time can be as long as $T_{1}$ time. This is due to the fact that an additional contribution to the $1 / T_{2}$ rate originating from the fluctuations of the spin-orbit-related magnetic field along the external magnetic field direction is proportional to the phonon density of states at zero frequency. Thus, this contribution is zero for acoustic phonons, see [22]. These results have motivated a whole variety of theoretical investigations on hyperfine interaction in quantum dots and related structures which will be reviewed in this paper [22-40].

A scenario similar to an electron bound in a quantum dot is the case of a shallow phosphorus donor in a silicon crystal, Si:P. This case is essential for the solid state quantum computing proposal by Kane [5]. The donor electron is bound to the $\mathrm{P}$ atom in a large hydrogen-like orbit with a Bohr radius of about $30 \AA$. The nuclear spins interacting with the electron are the central

${ }^{31} \mathrm{P}$ and the surrounding ${ }^{29} \mathrm{Si}$. The $T_{1}$ timescale for energy relaxation of the electron spin was 
determined by Feher and Gere [41] to be of the order of $10^{3} \mathrm{~s}$. The transverse spin decay was investigated by Gordon and Bowers [42] using the spin-echo method, deducing a timescale $T_{2}^{*}$ of the order of $500 \mu \mathrm{s}$, see also [43]. A very recent spin-echo study by Tyryshkin et al [44] on $\mathrm{P}$ donors in natural and isotopically purified $\mathrm{Si}$ has reported $T_{2}^{*}$ times being significantly larger than the previous result.

Finally we mention that hyperfine interaction between electron and nuclear spins is, of course, also investigated in higher-dimensional semiconductor nanostructures such as quantum wells. For recent work using optical NMR techniques we refer to [45, 46].

\section{Hyperfine interaction in semiconductors}

Hyperfine interaction is the coupling of a nuclear magnetic moment to the magnetic field provided by the (orbital and spin) magnetic moment of electrons. The Hamiltonian describing this interaction as a lowest-order relativistic correction was derived in 1930 by Fermi [47]. For an s-electron there is no orbital contribution and the Hamiltonian is $[47,48]$

$$
\mathcal{H}=\frac{4 \mu_{0}}{3 I} \mu_{\mathrm{B}} \mu_{I}\left|\psi\left(\vec{r}_{I}\right)\right|^{2} \vec{S} \vec{I}
$$

Here $\vec{S}$ is the spin of the electron and $\psi\left(\vec{r}_{I}\right)$ is its wavefunction at the location $\vec{r}_{I}$ of the nucleus. This Hamiltonian couples the electron spin to the nuclear spin $\vec{I}$ with total spin quantum number $I$ and magnetic moment $\mu_{I}$, which is represented by the operator $\vec{\mu}_{I}=\left(\mu_{I} / I\right) \vec{I}$. Both spin operators are taken to be dimensionless, $\mu_{0}=4 \pi \times 10^{-7} \mathrm{~V} \mathrm{~s} \mathrm{~A}^{-1} \mathrm{~m}^{-1}$ is the usual magnetic constant in SI units and $\mu_{\mathrm{B}}$ is the Bohr magneton. The leading contribution to the hyperfine coupling for higher angular momenta of the electron looks different from (1) and is essentially given by the usual dipolar coupling between the nuclear magnetic moment and the (orbital and spin) magnetic moment of the electron.

For the issue of spin coherence and interaction with nuclei in semiconductors, the most relevant case is electrons in s-type conduction bands. We shall therefore concentrate on this case where the hyperfine coupling is described by the Hamiltonian (1). From the viewpoint of nuclear magnetic resonance experiments this coupling is the origin of an increment in the position of resonance lines known as the Knight shift. From the viewpoint of electron spin resonance effects in solids this Hamiltonian describes the Overhauser field. As a general reference on both effects we refer the reader to the textbooks by Abragam [49] and Slichter [50].

In a semiconductor crystal the electron wavefunction is a product of a Bloch amplitude $u(\vec{r})$ and a modulating envelope function $\Psi(\vec{r}), \psi(\vec{r})=\Psi(\vec{r}) u(\vec{r})$. We therefore can rewrite the Hamiltonian as [48]

$$
\mathcal{H}=\frac{4 \mu_{0}}{3 I} \mu_{\mathrm{B}} \mu_{I} \eta\left|\Psi\left(\vec{r}_{I}\right)\right|^{2} \vec{S} \vec{I}
$$

with $\eta=\left|u\left(\vec{r}_{I}\right)\right|^{2}$. For free electrons the Bloch function is constant, $|u(\vec{r})|=1$; in a realistic crystal $|u(\vec{r})|$ has maxima at the lattice positions, i.e. the locations of the nuclei, leading to $\eta>1$. Values for $\eta$ in semiconductor systems can be estimated from electron spin resonance experiments [48]. For InSb Gueron [51] found $\eta_{\mathrm{In}}=6.3 \times 10^{3}, \eta_{\mathrm{Sb}}=10.9 \times 10^{3}$; for GaAs Paget et al [52] estimated $\eta_{\mathrm{Ga}}=2.7 \times 10^{3}, \eta_{\mathrm{As}}=4.5 \times 10^{3}$. From NMR experiments on ${ }^{29} \mathrm{Si}$ Shulman and Wyluda estimated a value of $\eta_{\mathrm{Si}}=186$ [53]. 


\section{Electron spin dynamics in quantum dots due to hyperfine interaction with nuclei}

\subsection{Modelling hyperfine interaction in quantum dots}

We consider an electron confined in a semiconductor quantum dot in an s-type conduction band. We assume the electron to be in some orbital eigenstate according to the confining potential, e.g. the orbital ground state in the quantum dot. The remaining spin degree of freedom is coupled to an external magnetic field $\vec{B}$ with an electronic $g$ factor $g$, and to the spins of surrounding nuclei via the hyperfine contact interaction described in the previous section. Thus the Hamiltonian is

$$
\mathcal{H}=g \mu_{\mathrm{B}} \vec{S} \vec{B}+\vec{S} \sum_{i} A_{i} \vec{I}_{i}
$$

Here the subscript $i$ labels the nuclei and the coupling constants $A_{i}$ are given by (cf (2))

$$
A_{i}=A v_{0}\left|\Psi\left(\vec{r}_{i}\right)\right|^{2}
$$

with

$$
A=\frac{4 \mu_{0}}{3 I} \mu_{\mathrm{B}} \mu_{I} \eta n_{0}
$$

where $n_{0}=1 / v_{0}$ is the density of the nuclei. Provided that the electronic envelope wavefunction $\Psi(\vec{r})$ varies smoothly on the length scale given by $\sqrt[3]{v_{0}}$ it is appropriate to replace the sum $\sum_{i} A_{i}$ by an integral over space; then $A=\sum_{i} A_{i}$ up to small corrections to this approximation. The Hamiltonian (3) also describes the hyperfine interaction between nuclear spins and the spin of an electron bound in a hydrogen-type orbit around a phosphorus donor in a silicon crystal.

Let us now address the order of magnitude of the hyperfine interaction. A GaAs quantum dot with a volume of the order of $10^{5} \mathrm{~nm}^{3}$ contains of the order of $N=10^{6}$ nuclei with a density $n_{0}=45.6 \mathrm{~nm}^{-3}$. Taking into account the natural abundances of the three occurring isotopes ${ }^{69} \mathrm{Ga},{ }^{71} \mathrm{Ga}$ and ${ }^{75} \mathrm{As}$ one has an average nuclear magnetic moment of $\mu_{I}=1.84 \mu_{\mathrm{N}}$. With the values for $\eta$ estimated in [52] this leads to an overall coupling constant $A$ of the order of $10^{-5}-10^{-4} \mathrm{eV}$. This is the strength of the hyperfine coupling acting on the electron spin in the presence of a fully polarized nuclear spin system. With the effective electron $g$ factor $g=-0.44$ for GaAs this corresponds to an effective magnetic field of the order of a few tesla. For a completely unpolarized nuclear spin system the strength of the hyperfine field is fluctuating around zero with a typical value given by $A / \sqrt{N}$, where $N$ is the number of nuclei in the dot effectively interacting with the electron spin. Note that for GaAs all nuclear magnetic moments are positive, leading to an antiferromagnetic sign for the hyperfine coupling. This is different from the situation in Si:P where the magnetic moment of ${ }^{31} \mathrm{P}$ is positive while the surrounding ${ }^{29} \mathrm{Si}$ have a negative nuclear magnetic moment, resulting in a dominantly ferromagnetic coupling to the electron spin. The natural abundance of ${ }^{29} \mathrm{Si}$ leads to a density $n_{0}=2.3 \mathrm{~nm}^{-3}$. Assuming a Bohr radius of $30 \AA$ for the hydrogen-like electron orbit one can estimate the number of nuclei effectively interacting with the electron spin to be of the order of a few hundred and from (5) one finds values of $|A|$ of the order of $10^{-7} \mathrm{eV}$ using the estimate for $\eta$ given in [53].

Another kind of interaction in the systems discussed above is the dipolar coupling between nuclear spins. This contribution to the Hamiltonian is obtained from its classical counterpart by expressing the magnetic moments in terms of nuclear spin operators, $\vec{\mu}_{I}=\left(\mu_{I} / I\right) \vec{I}$. Thus the interaction between two nuclear spins labelled by 1 and 2 is

$$
\mathcal{H}_{12}=-\frac{\mu_{0}}{4 \pi} \frac{\mu_{1} \mu_{2}}{I_{1} I_{2}} \frac{1}{r_{12}^{3}}\left(\frac{3\left(\vec{I}_{1} \vec{r}_{12}\right)\left(\vec{I}_{2} \vec{r}_{12}\right)}{r_{12}^{2}}-\vec{I}_{1} \vec{I}_{2}\right)
$$


where $\vec{r}_{12}$ is the distance vector between the nuclei. In $\mathrm{GaAs}{ }^{71} \mathrm{Ga}$ has the largest nuclear magnetic moment with $\mu_{I}=2.562 \mu_{\mathrm{N}}$. Assuming two of such nuclei being on nearestneighbour sites of the underlying zinc-blende lattice one finds $r_{12}=0.24 \mathrm{~nm}$ and the energy scale of the dipolar interaction between these two nuclei has the value $\left(\mu_{0} / 4 \pi\right) \mu_{I}^{2} / r_{12}^{3}=$ $7.6 \times 10^{-12} \mathrm{eV}$. This is an upper bound for the dipolar coupling between neighbouring nuclei in GaAs and sets the timescale $\left(10^{-4}-10^{-5} \mathrm{~s}\right)$ on which this interaction indirectly influences the quantum dynamics of the electron spin. As we shall see below, the largest timescales relevant for electron spin dynamics due to hyperfine coupling are of the order of $10^{-6} \mathrm{~s}$. Therefore, the timescale of the dipolar coupling is much larger than the timescale provided by the hyperfine interaction; analogous considerations can be made for the case of Si:P. In the following we shall therefore neglect the dipolar interaction unless stated otherwise. A contribution to the Hamiltonian which we also neglect is the coupling of the nuclear spins to the external magnetic field. This interaction is much smaller than the Zeeman coupling of the electron spin because of the smallness of the nuclear magneton compared with the Bohr magneton for electrons.

4.1.1. Ground state and elementary properties. If the sign of the hyperfine interaction is ferromagnetic ( $A<0$, as realized by the coupling to ${ }^{29} \mathrm{Si}$ nuclear spins) the ground state of the Hamiltonian (3) is (for $\vec{B}=0$ ) the fully spin-polarized multiplet with all (electron and nuclear) spins in parallel, resulting in the maximum value of the total spin quantum number, $J=N I+1 / 2$. For an antiferromagnetic sign the classical ground state (neglecting the operator nature of spins) has all nuclear spins in parallel and the electron spin pointing opposite to them. We therefore anticipate that the true quantum mechanical ground state will have (again for vanishing external magnetic field) the total spin quantum number $J=N I-1 / 2$. This assumption is confirmed by our numerics. Figure 1 shows the energy spectrum of the Hamiltonian (3) for zero magnetic field as a function of the total spin quantum number $J$ in a system of $N=13$ nuclear spins of length $I=1 / 2$. Clearly the ground state lies in the subspace of $J_{z}=(13-1) / 2=6$. A general state in this subspace can be written as

$$
|\psi\rangle=\alpha S^{-}|\Uparrow, \uparrow \cdots \uparrow\rangle+\sum_{i} \frac{\beta_{i}}{\sqrt{2 I}} I_{i}^{-}|\uparrow, \uparrow \cdots \uparrow\rangle
$$

where $i$ labels the nuclei, $|\uparrow, \uparrow \cdots \uparrow\rangle$ is the fully spin-polarized ground state with all spins pointing upward and we have introduced the usual spin lowering operators for the electron spin of length $1 / 2$ and nuclear spins of length $I$. The stationary Schrödinger equation leads to the following system of equation for the amplitudes $\alpha$ and $\beta_{i}$ :

$$
\begin{aligned}
& -\left(\frac{I}{2} A+\frac{\varepsilon_{z}}{2}+E\right) \alpha+\sum_{i} \sqrt{\frac{I}{2}} A_{i} \beta_{i}=0 \\
& \sqrt{\frac{I}{2}} A_{i} \alpha+\left(\frac{I}{2}\left(A-2 A_{i}\right)+\frac{\varepsilon_{z}}{2}-E\right) \beta_{i}=0 .
\end{aligned}
$$

Here $E$ is the energy eigenvalue and we have reintroduced a finite Zeeman coupling $\varepsilon_{z}=g \mu_{\mathrm{B}} B$. From the second of the above equations one finds

$$
\beta_{i}(E)=\frac{\sqrt{I / 2} A_{i} \alpha(E)}{E-\left(I\left(A-2 A_{i}\right)+\varepsilon_{z}\right) / 2} .
$$

The ground state energy for $\left|\varepsilon_{z}\right| \ll A$ is of the order of $E \approx-A I / 2$. Thus the denominator of the rhs of (10) is of the order of $A$. Since the couplings $A_{i}$ are of the order of $A / N$ we see that each $\beta_{i}$ is smaller than $\alpha$ by a factor of the order of $1 / N$. Therefore $|\alpha| \approx 1$ up to quantum corrections and all coefficients $\beta_{i}(E)$ are of the order of $1 / N$. Thus, the corrections to the classical ground state $(|\alpha|=1)$ are of the order of $1 / N,|\alpha|^{2}=1-\sum_{i}\left|\beta_{i}\right|^{2} \approx 1-1 / N$. 


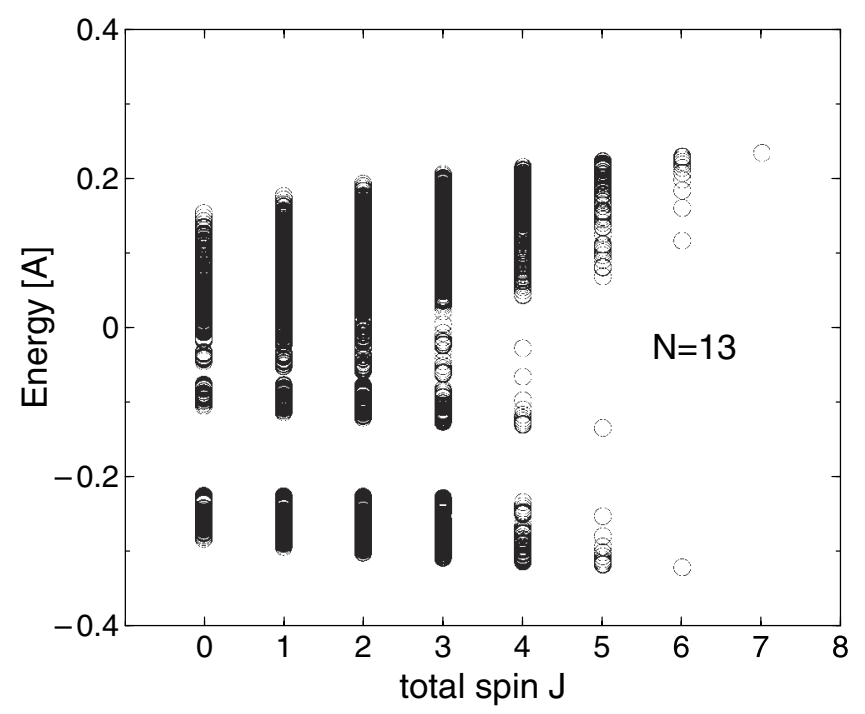

Figure 1. Energy spectrum (in units of $A>0$ ) of the Hamiltonian (3) for zero magnetic field as a function of the total spin quantum number $J$ in a system of $N=13$ nuclear spins of length $1 / 2$. For details about the modelling of coupling constants see section 4.3.1.

In summary, for large systems $(N \gg 1)$, the ground state is essentially given by a tensor product state with all nuclear spins in parallel and the electron spin pointing opposite to them. Excited states with the same total spin quantum number along the direction of the nuclear polarization are separated from the ground state by a substantial gap of the order of the coupling parameter $A$.

These results were obtained recently by Khaetskii et al [24] who studied the time evolution of the classical ground state under the quantum Hamiltonian (3). These investigations were carried out using the standard Laplace transform technique. A detailed account of the mathematical details of this approach has been given recently in [25]. The main findings are the following. The classical ground state for $\left|\varepsilon_{z}\right| \ll A$ remains constant in time up to quantum corrections of the order of $1 / N$. Starting the time evolution at $t=0$ with $|\alpha(t=0)|=1$ the electron spin expressed in terms of its expectation value $\left\langle S^{z}(t)\right\rangle$ undergoes coherent oscillations between $\left\langle S^{z}(t)\right\rangle=-1 / 2$ and $\left\langle S^{z}(t)\right\rangle=-1 / 2+\mathcal{O}(1 / N)$ with a period of $T=4 \pi \hbar / A$ over a timescale of the order of $\hbar N / A$. This timescale is nothing other than the characteristic period of precession of an individual nuclear spin in the field generated by the electron spin. At this timescale a different nuclear spin configuration is created and, because of the spatial variation of the hyperfine coupling constants inside the dot, this leads to a different random value of the nuclear field seen by the electron spin and thus to its decoherence. After a time interval of this order the oscillations fade out and the expectation value $\left\langle S^{z}(t)\right\rangle$ decays to a value of the order of $\left\langle S^{z}(t \rightarrow \infty)\right\rangle=-1 / 2+\mathcal{O}(1 / N)$, see figure 2 .

Thus the decaying part of the initial spin state has smallness $1 / N$ which is due to a large gap $\simeq A$ seen by the electron spin through the hyperfine interaction for a fully polarized state. As a result, only a small portion $\sim 1 / N$ of the opposite $(+1 / 2)$ state can be admixed. Moreover, the decay of the electron spin turns out to be non-exponential for any external magnetic field. In the case when the perturbative treatment is applicable (i.e. a large Zeeman field) it follows a power law with a leading term proportional to $t^{-3 / 2}$. The fact that the decay of the electron spin is not exponential can be easily understood. The exponential decay occurs when the correlation time of the randomly fluctuating field which causes the decoherence is short compared to the decoherence time. As a result the Markovian approximation can be applied. In our case 


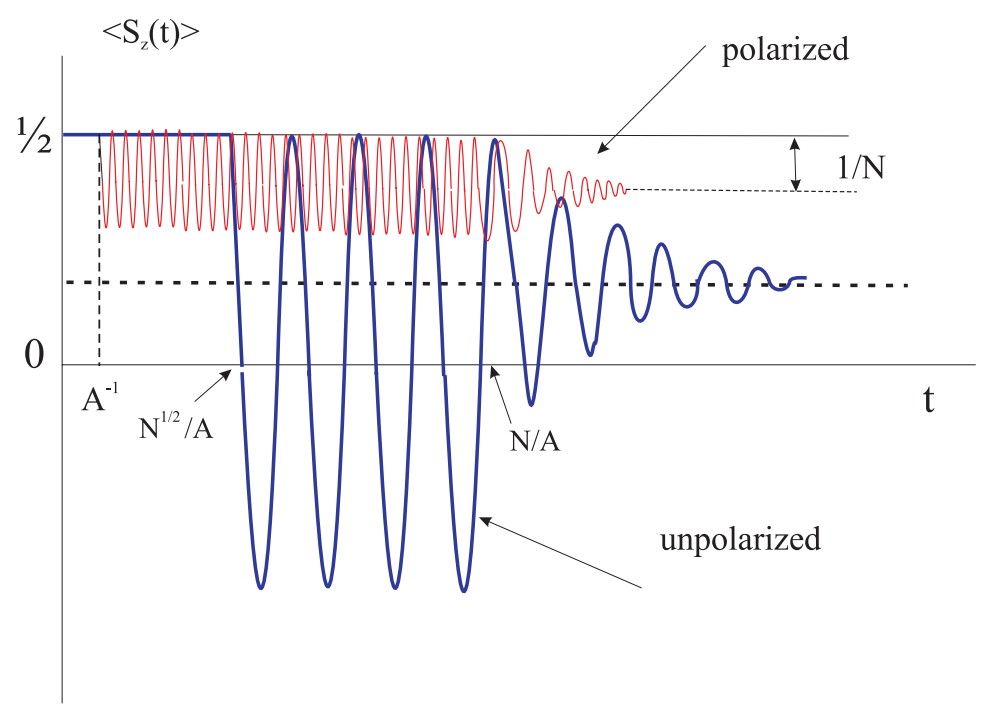

Figure 2. Schematic dependence of $\left\langle S_{z}(t)\right\rangle$ on time $t$ for the unpolarized tensor product and fully polarized nuclear states. The timescale for the onset of the decay $\sim N / A$ is the same for both cases. In the fully polarized case the magnitude of the effect is $1 / N$. The period of oscillations is of the order of $\sqrt{N} / A$ for the unpolarized and $\sim 1 / A$ for the polarized case.

(This figure is in colour only in the electronic version)

the non-exponential behaviour is a result of the fact that the correlation time for the nuclear magnetic field seen by the electron spin is itself determined by the flip-flop processes since the internal nuclear dynamics is excluded. Thus the Markovian approximation is not valid, see for comparison section 4.6.2.

A particular situation arises in the time evolution of the state (7) with initially $\mid \alpha(t=$ $0) \mid=1$ if an external Zeeman field is applied to the electron spin which approximately cancels the initial Overhauser field, i.e. $\varepsilon_{z} \approx-A I$ in the above conventions [24]. Near this Zeeman field $|\alpha|^{2}$ averaged over time is $1 / 2$, i.e. the up and down states of the electron spin are strongly coupled via the nuclei (see figure 3 ). In contrast, outside this resonance regime the value of $|\alpha|^{2}$ is close to unity (with small $1 / N$ corrections), i.e. $\left\langle S^{z}(t)\right\rangle=1 / 2-|\alpha|^{2}$ is close to $-1 / 2$ at any time. The width of the resonance is $\sim A / \sqrt{N}$, i.e. small compared to the initial gap $A I$. This abrupt change in the amplitude of oscillations of $\left\langle S^{z}(t)\right\rangle$ (when changing $\varepsilon_{z}$ in a narrow interval around $A I$ ) can be used for an experimental detection of the fully polarized state.

Although physically not particularly realistic, it is also instructive to study the model (3) in the case of all coupling constants being equal to each other, $A_{i}=A / N$ for all $i$. Models of this type were studied recently by Eto [33] and Semenov and Kim [34], see also [25]. The technical advantage of this type of model lies in the fact that the square of the total nuclear spin $\vec{I}_{\text {tot }}=\sum_{i} \vec{I}_{i}$ is an additional conserved quantity, $\left[\mathcal{H}, I_{\text {tot }}^{2}\right]=0$. If $A_{i}=A / N$ the Hamiltonian in the absence of an external magnetic field is

$$
\mathcal{H}=\frac{A}{2 N}\left(\left(\vec{I}_{\text {tot }}+\vec{S}\right)^{2}-\vec{I}_{\text {tot }}^{2}-\vec{S}^{2}\right) .
$$

Since the total spin $\vec{J}=\vec{I}_{\text {tot }}+\vec{S}$ can have values $J=I_{\text {tot }} \pm 1 / 2$, each value of the quantum number $I_{\text {tot }}$ corresponds to two energy levels given by

$$
E(I)=\frac{A}{2 N}\left( \pm\left(I_{\mathrm{tot}}+\frac{1}{2}\right)-\frac{1}{2}\right) .
$$




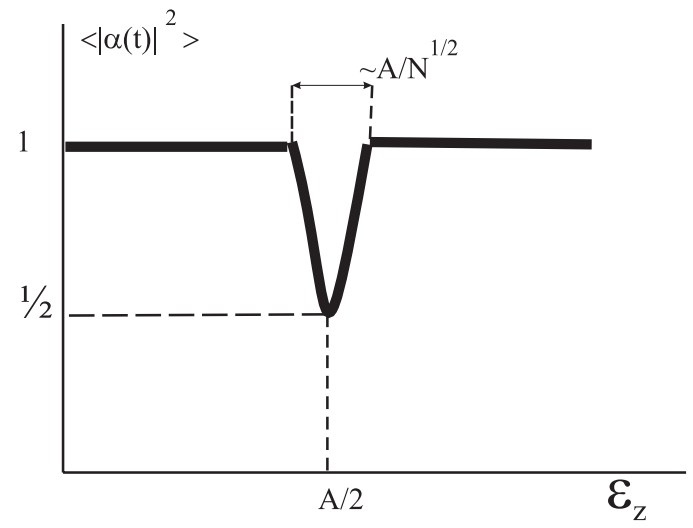

Figure 3. The dependence of $|\alpha|^{2}$ averaged over time $\left(\left\langle|\alpha(t)|^{2}\right\rangle\right)$ on the external Zeeman field $\epsilon_{z}$ for a fully polarized nuclear state. The resonance occurs at $\left|\epsilon_{z}\right|=A / 2$ and the width of the resonance is $\sim A / \sqrt{N}$, which is much smaller than the initial gap $A / 2$.

These energy levels are typically highly degenerate. For instance, if the nuclear spins are of length $1 / 2$, the different values of $I_{\text {tot }}$ occur with a degeneracy of

$$
\left(\begin{array}{c}
N \\
N / 2-I_{\text {tot }}
\end{array}\right)-\left(\begin{array}{c}
N \\
N / 2-I_{\text {tot }}-1
\end{array}\right) \text {. }
$$

As seen from equation (12), the spectrum is equidistant with a level spacing $\Delta E=A / 2 N$. Therefore the time evolution of an arbitrary state is strictly periodic with a recurrence time $T=4 \pi \hbar N / A$. However, in contrast to naive expectations, even in this simple case there is some time dependence of $\left\langle S^{z}(t)\right\rangle$ which cannot be described by a single frequency. Actually, for a nuclear state with given $I_{\text {tot }}^{z}$ the solution contains all the frequencies of the form $\Delta E=A\left(2 I_{\text {tot }}+1\right) / 2 N$, where $I_{\text {tot }}$ are all the moduli of the total nuclear momentum which can have this projection, $I_{\text {tot }}^{z}$.

Depending on the number and length of the nuclear spins, this can lead to shorter periodicities in the time evolution of $\langle\vec{S}(t)\rangle$. For instance, for an odd number of half-integer nuclear spins $2 I_{\mathrm{tot}}+1$ is even and $\langle\vec{S}(t)\rangle$ has a period of $T=2 \pi \hbar N / A$.

4.1.2. Integrability. If all nuclear spins are of length $1 / 2$, the spin Hamiltonian (3) has the strong mathematical property of being integrable and solvable via an appropriate version of the Bethe ansatz. This fact was recognized first by Gaudin in a rather formal context [54]. Consider a system of $N$ spins $1 / 2$ and the following sequence of operators [54, 55]:

$$
\mathcal{H}_{i}=\sum_{j \neq i} \frac{\vec{\sigma}_{i} \vec{\sigma}_{j}}{z_{i}-z_{j}}
$$

where $\vec{\sigma}_{i}$ are Pauli matrices and the $z_{i}$ are some arbitrary coupling parameters. Obviously, by fixing one spin $i$ to be the central spin and adjusting the couplings to the other spins, the operator $\mathcal{H}_{i}$ assumes the form of the Hamiltonian (3). The operators $\mathcal{H}_{i}$ commute with each other:

$$
\left[\mathcal{H}_{i}, \mathcal{H}_{j}\right]=0,
$$

and fulfil the sum rule

$$
\sum_{i} \mathcal{H}_{i}=0 .
$$


Thus, $N-1$ of these operators together with the square of the total spin form a set of $N$ linearly independent commuting operators being bilinear in the individual spin operators. The coordinate Bethe ansatz diagonalizing simultaneously all $\mathcal{H}_{i}$ can be summarized as follows $[54,55]$. Consider states of the form

$$
\left|w_{1}, \ldots, w_{m}\right\rangle=F\left(w_{1}\right) \cdots F\left(w_{m}\right)|\uparrow \cdots \uparrow\rangle,
$$

where $|\uparrow \cdots \uparrow\rangle$ is the spin-polarized state with all spins in parallel, and

$$
F(w)=\sum_{i} \frac{\sigma_{i}^{-}}{w-z_{i}}
$$

with $\sigma_{i}^{-}=\sigma_{i}^{x}-\mathrm{i} \sigma_{i}^{y}$. These states are eigenstates of all $\mathcal{H}_{i}$ if and only if the complex parameters $w_{1} \ldots w_{m}$ fulfil the Bethe equations

$$
\sum_{i=1}^{N} \frac{1}{w_{k}-z_{i}}+\sum_{\substack{l=1 \\ l \neq k}}^{m} \frac{2}{w_{l}-w_{k}}=0 .
$$

For a given solution of these equations the corresponding eigenvalues are

$$
\varepsilon_{i}\left(w_{1}, \ldots, w_{m}\right)=\sum_{l=1}^{m} \frac{2}{w-z_{i}}+\sum_{j \neq i} \frac{1}{z_{i}-z_{j}} .
$$

The eigenstates (17) are states of the highest weight in multiplets of the total spin with respect to its $z$ component. States lower in these multiplets can be obtained by applying the lowering operator of the total spin, or by formally considering solutions to the Bethe equations (19) with some $w_{k}$ being infinite. By counting the number of solutions of (19) it can be shown that the Bethe ansatz produces all multiplets, i.e. all energy eigenvalues.

The coordinate Bethe ansatz outlined above has been extended to the technique of the algebraic Bethe ansatz [56]. We also note that equations (19) are a limiting case of the Bethe equations of the so-called Richardson model describing electron pairing in superconducting grains [57]. This issue has attracted considerable interest recently [58-61] and an algebraic version of the Bethe ansatz has also been presented [60].

For practical purposes, the treatment of the Bethe equations (19) is still rather complicated and explicit results, for instance for correlation functions, are difficult to obtain [62]. In the remainder of this review we shall therefore concentrate on techniques other than the Bethe ansatz. It is an interesting and important question to what extent certain results, such as the non-exponential spin decay, depend on the integrability of the model (3). We stress that this integrability holds very generally and does not depend on a specific choice of the hyperfine coupling constants $A_{i}$.

\subsection{Different types of initial states}

In the numerical simulations to be described below the electron spin is initially in a single tensor product state with the nuclear spin system

$$
|\psi(t=0)\rangle=\left|\psi_{\mathrm{el}}\right\rangle \otimes\left|\psi_{\mathrm{nuc}}\right\rangle
$$

i.e. the electron spin described by $\left|\psi_{\mathrm{el}}\right\rangle$ is initially uncorrelated with the nuclear spins. However, there are still quite a variety of possibilities for the initial nuclear spin state $\left|\psi_{\text {nuc }}\right\rangle$. A simple choice would be just a tensor product of eigenstates with respect to a given quantization axis, say, the $z$ direction:

$$
\left|\psi_{\text {nuc }}\right\rangle=|\uparrow\rangle_{1} \otimes|\downarrow\rangle_{2} \otimes|\downarrow\rangle_{3} \otimes \cdots \otimes|\uparrow\rangle_{\mathrm{N}}
$$


where we have for simplicity assumed the nuclear spins to be of length $1 / 2$. A nuclear spin state close to the above form can be generated experimentally by cooling down the nuclear spins in a strong external magnetic field. The strong magnetic field provides a quantization axis and suppresses dipolar interactions changing the spin projection along the field axis. Then, due to spin-lattice relaxation processes, the nuclear spin system will end up in a state of the type (22).

A more general possibility of a nuclear spin state is a tensor product state but with individual polarization directions for each spin:

$$
\left|\psi_{\text {nuc }}\right\rangle=\left(a_{1}|\uparrow\rangle_{1}+b_{1}|\downarrow\rangle_{1}\right) \otimes \cdots \otimes\left(a_{N}|\uparrow\rangle_{N}+b_{N}|\downarrow\rangle_{N}\right)
$$

where the complex numbers $a_{i}, b_{i}$ parametrize the spin state and are subject to the normalization condition $\left|a_{i}\right|^{2}+\left|b_{i}\right|^{2}=1$. A yet more general state of the nuclear spins is a superposition of tensor product states:

$$
\left|\psi_{\text {nuc }}\right\rangle=\sum_{T} \alpha_{T}|T\rangle
$$

where the summation runs over all tensor product states of the form (22), i.e. over a complete basis of the underlying Hilbert space. If one works in a subspace corresponding to a given value of $J^{z}$, this summation has to be restricted accordingly. For a nontrivial choice of the amplitudes $\alpha_{T}$ states of the above form can, in general, not be expressed as tensor product states, whatever basis one would choose in the space of each nuclear spin. Therefore, such states are, in general, correlated or, using the language of quantum information theory, entangled [63, 64]. A particular class of correlated nuclear states is obtained when the complex amplitudes $\alpha_{T}$ are chosen at random, only restricted by the normalization condition $\sum_{T}\left|\alpha_{T}\right|^{2}=1$. In the following we will refer to this type of states as randomly correlated states. As we shall see, the type of initial state of the nuclear spin system has a profound impact on the electron spin dynamics.

\subsection{Numerical results for electron spin dynamics: product states versus randomly correlated states}

Here we review our recent numerical studies [32] of electron spin dynamics in quantum dots modelled by the Hamiltonian (3). These investigations are based on exact numerical diagonalizations whose technical details we summarize below.

4.3.1. Numerical method and modelling. Our simulations of the electron spin dynamics are performed by numerically diagonalizing the Hamiltonian (3). The numerical diagonalization makes use of the fact that the projection of the total spin $\vec{J}=\vec{S}+\sum_{i} \vec{I}_{i}$ on the direction of the external field (usually chosen as the $z$ axis) is a conserved quantity leading to a block-diagonal structure of the Hamiltonian matrix. Therefore it is convenient to work in subspaces of a given value of $J^{z}$. The Hamiltonian is then diagonalized within such a subspace and the time evolution of a given initial state is obtained from the eigensystem data. For a numerically exact simulation of the time evolution of a general initial state one generally needs the full eigensystem, i.e. all eigenvalues and corresponding eigenvectors. If the initial state involves more than one of the above invariant subspaces of the Hamiltonian the time evolution in the different subspaces can be superimposed. We note that this method of solving for the quantum mechanical time evolution is non-iterative and can therefore be extended to very large times. On the other hand it requires the full eigensystem in a given invariant subspace of the Hamiltonian and it is the dimensions of these subspaces that limit our numerical investigations. To reduce the numerical demands we will consider in the following nuclear spin of length $I=1 / 2$. The 
dimensions of the invariant subspaces increase with decreasing $J^{z}$, starting from the maximum value $J^{z}=(N+1) / 2$ according to a binomial distribution, where $N$ is the number of nuclei considered. The dimensions of the invariant subspaces become largest for the minimum value of $\left|J^{z}\right| \in\{0,1 / 2\}$. For the latter case we could simulate the full time evolution for systems with up to 14 nuclear spins.

In our simulations we will assume a spherical quantum dot using the following specific modelling. A given number $N$ of nuclear spins is contained in a sphere of radius

$$
R=\left(\frac{3 N}{4 \pi n_{0}}\right)^{1 / 3}
$$

where $n_{0}=1 / v_{0}$ is the density of nuclei. The electron wavefunction is given by

$$
|\Psi(\vec{r})|^{2}=\left(\frac{1}{\pi(R / a)^{2}}\right)^{3 / 2} \mathrm{e}^{-r^{2} /(R / a)^{2}}
$$

where the parameter $a$ describes the confinement of the electron in the dot due to an essentially harmonic potential (with possibly small anharmonic corrections). In the following we shall use $a=2$ such that the electron is reasonably confined in the sphere of radius $R$. The question of different types of confining potentials was investigated in [30]. Moreover, we shall use the material parameters of GaAs with $n_{0}=45.6 \mathrm{~nm}^{-3}$. Therefore a typical quantum dot contains about $N=10^{5}$ nuclei. To mimic their spherical distribution also in systems of smaller size used in our simulations we choose the radial coordinate $r_{i}$ of the $i$ th nucleus according to

$$
r_{i}=\left(\frac{3(i-(1 / 2))}{4 \pi n_{0}}\right)^{1 / 3}
$$

with $i$ ranging from 1 to $N$. The results to be presented below are obtained for an antiferromagnetic sign of the hyperfine coupling, $A>0$.

4.3.2. Results for electron spin dynamics. Figure 4 shows results for a system of $N=14$ nuclear spins. This is the largest system size for which we have been able to treat the electron spin dynamics in the presence of an unpolarized nuclear system. The upper left panel shows the expectation value $\left\langle S^{z}(t)\right\rangle$ as a function of time for an initially fully polarized nuclear system with the electron spin pointing opposite to it in the negative $z$ direction. In the following panels the polarization of the nuclear system is successively reduced by lowering the value of $J^{z}$ in the initial state. The case of a fully unpolarized nuclear spin system is reached in the bottom right panel with $J^{z}=-1 / 2$. Since the value of the $z$ component of the total spin $J$ is fixed the expectation values of the transversal components $\left\langle S^{x}\right\rangle$ and $\left\langle S^{y}\right\rangle$ vanish. In all simulations shown in figure 4 the electron spin is initially in a tensor product state with the nuclear system. The nuclear spins themselves are initially in a randomly correlated state as described in section 4.2.

In all cases, $|\langle\vec{S}(t)\rangle|=\left|\left\langle S^{z}(t)\right\rangle\right|$ decreases in magnitude. With decreasing polarization the decay becomes more pronounced and the oscillations accompanying this process get suppressed. Note that it is the decay of the envelope in these graphs, but not the fast oscillations themselves, that signals the decay of the spin. The distance between two neighbouring maxima of the oscillations can depend slightly on the initial state and the coupling constants in the Hamiltonian. However, a good estimate for this effective period is usually given by $T=4 \pi \hbar / A$, since $A / 2$ is an estimate (neglecting quantum fluctuations) for the width of the spectrum, i.e. the difference between the largest and the smallest eigenvalue of the Hamiltonian.

When the nuclear spin system is initially in a randomly correlated state the time evolution of $\left\langle S^{z}(t)\right\rangle$ is very reproducible, in the sense that it depends only very weakly on the particular 


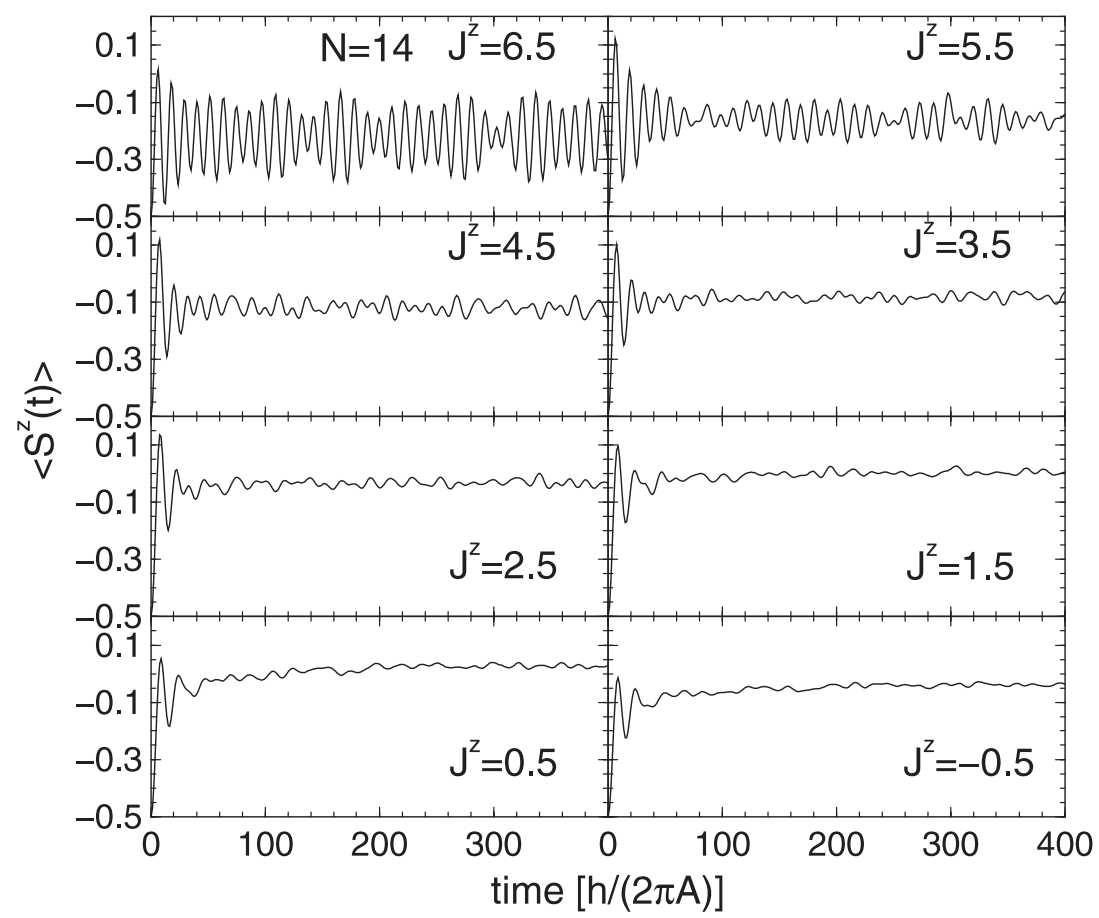

Figure 4. The time evolution of the electron spin in a system of $N=14$ nuclear spins of length $1 / 2$ for different degrees of polarization of the randomly correlated nuclear system. The hyperfine coupling constants are induced by the quantum dot geometry. In all simulations the electron spin is initially pointing downward in a tensor product with the nuclear system. In the top left panel the nuclear spins are fully polarized in the initial state with the electron spin pointing opposite to them $\left(J^{z}=13 / 2\right)$. In the following panels the number of flipped nuclear spins in the initial state is gradually increased. The case of an initially fully unpolarized (but randomly correlated) nuclear system is reached in the bottom right panel $\left(J^{z}=-1 / 2\right)$. Here and in the following we take spins to be dimensionless, i.e. measured in units of $\hbar$.

representation of the initial random state. This is illustrated in figures 5 and 6 where the results of different randomly correlated initial nuclear spin states are compared for two different system sizes and degrees of polarization.

This behaviour of randomly correlated initial states sharply contrasts with the time evolution of a simple tensor product nuclear spin state. Figure 7 shows the time evolution of the electron spin for two initial tensor product states for the same system size and degree of polarization as in figure 6 . The comparison of these two figures demonstrates the significant difference in the electron spin dynamics for these two types of initial conditions in the nuclear system. In the case of tensor product initial states the time evolution depends significantly on the concrete initial condition and the decay of the electron spin occurs typically more slowly than in the case of an initially randomly correlated nuclear spin system.

4.3.3. Spin decay and quantum parallelism. In the two left panels of figure 8 we show the time evolution of the electron spin averaged over all nuclear tensor product states for two different system sizes and initial polarizations of the nuclear spins. The two right panels show the corresponding data for a single randomly correlated initial nuclear state. Comparing those plots one sees that these data are very close to the time evolution of a randomly correlated state. 


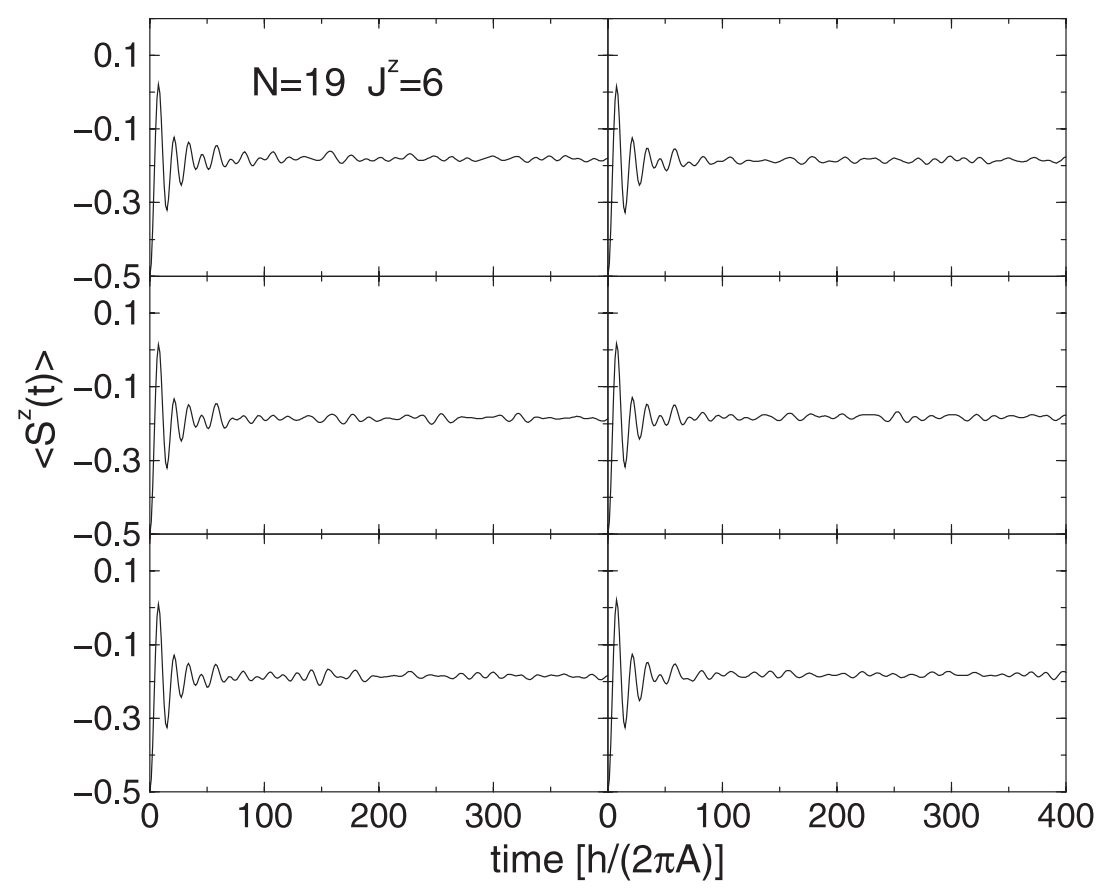

Figure 5. Electron spin dynamics for an initially randomly correlated nuclear spin system for $N=19$ nuclear spins with a moderate degree of polarization $\left(J^{z}=6\right)$. The data for six completely independent random realizations of the initial nuclear spin state are shown. The resulting electron spin dynamics is practically independent of the realization of the initial state for this type of initial condition.

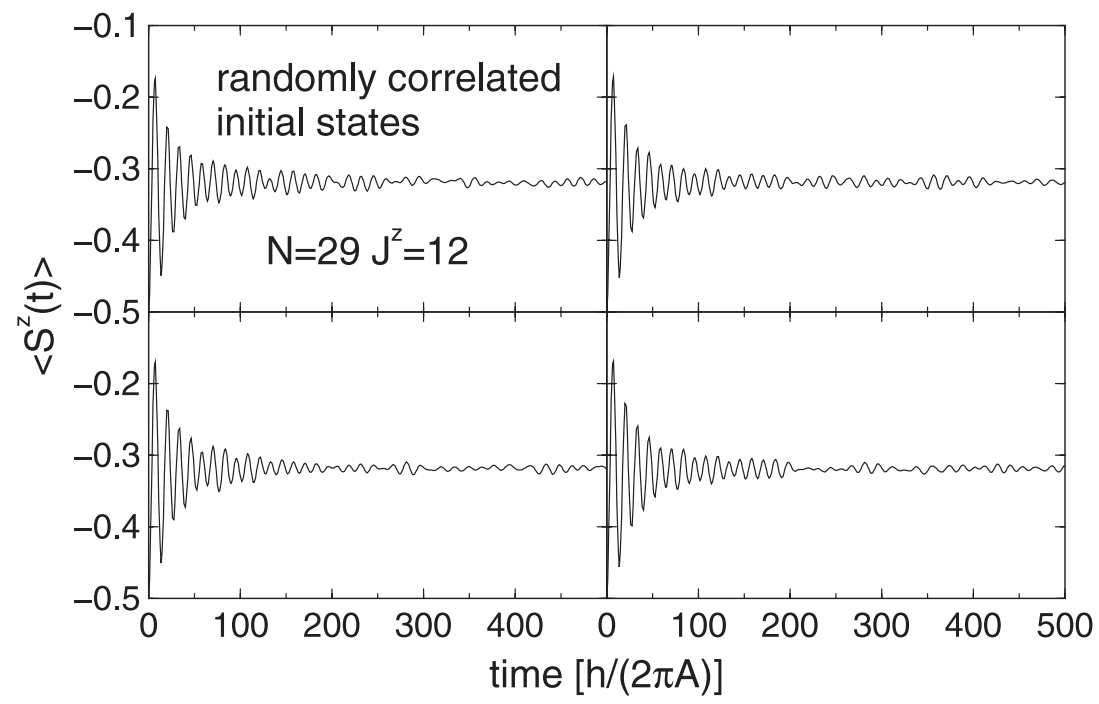

Figure 6. Data of the same type as in figure 5 for $N=29$ nuclei and $J^{z}=12$. Again, the electron spin dynamics is practically independent of the realization of the random initial nuclear state.

This observation is also made for other system sizes and degrees of polarization and constitutes an example of quantum parallelism [64]: the time evolution of each initially uncorrelated 


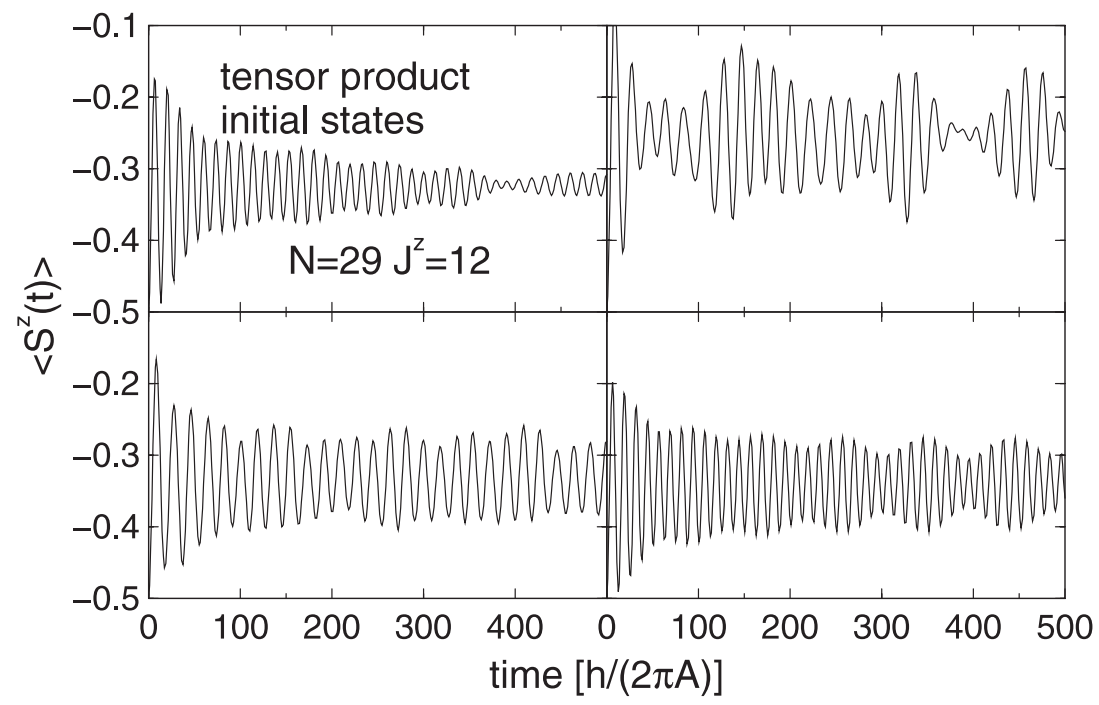

Figure 7. Electron spin dynamics for $N=29$ nuclei and $J^{z}=12$. Here the initial state of the nuclear spins is given by individual tensor product states. Different initial tensor product states clearly lead to a significantly different time evolution of the electron spin. As seen in figure 6 this is strikingly different from randomly correlated initial conditions.

(and therefore classical-like) nuclear state is present in the evolution of a linear superposition of all such states. In other words, the time evolutions of all uncorrelated classical-like states are performed in parallel in the time evolution of the randomly correlated state. An experimental consequence of this observation is that, if the electron spin dynamics would be detected on an array of independent quantum dots, one could not distinguish whether the nuclear spin system in each dot was initially randomly correlated or in an uncorrelated tensor product state. In other words, the spin dynamics of a randomly correlated pure state of the nuclear system in a single dot cannot be distinguished from a mixed state of an ensemble of dots.

As seen above, for randomly correlated initial nuclear states the time evolution of the electron spin does not practically depend on the concrete realization of the random nuclear state and mimics closely the average over all tensor product initial conditions. This observation relies on the cancellation of off-diagonal terms $\alpha_{T}^{*} \alpha_{T^{\prime}}\left\langle\Downarrow, T|\vec{S}(t)| \Downarrow, T^{\prime}\right\rangle, T \neq T^{\prime}$, due to the randomness in the phases of the coefficients $\alpha_{T}$. In this sense our system has a self-averaging property. This can be checked explicitly by reducing this randomness. The left panel of figure 9 shows the time evolution of a randomly correlated state where the amplitudes $\alpha_{T}$ are restricted to have a non-negative real and imaginary part. This time evolution turns out to be similarly reproducible as before, i.e. it does not depend on the concrete realization of the initial random state, but it is clearly different from the former case since the cancellation of off-diagonal contributions is inhibited. For comparison we show in the right panel of figure 9 data where the amplitudes in the initial nuclear spin state have a random phase but are restricted to have the same modulus. Here the proper averaging process takes place again.

The results described so far were obtained in certain subspaces of $J^{z}$ and for the form of coupling constants $A_{i}$ as induced by the quantum dot geometry. However, our findings do not depend on these choices. We have also performed simulations where the initial state has an overlap in the full Hilbert space. For a randomly correlated initial nuclear spin state the only difference is that now transverse components $\left\langle S^{x}(t)\right\rangle,\left\langle S^{y}(t)\right\rangle$ of the electron spin also evolve. 


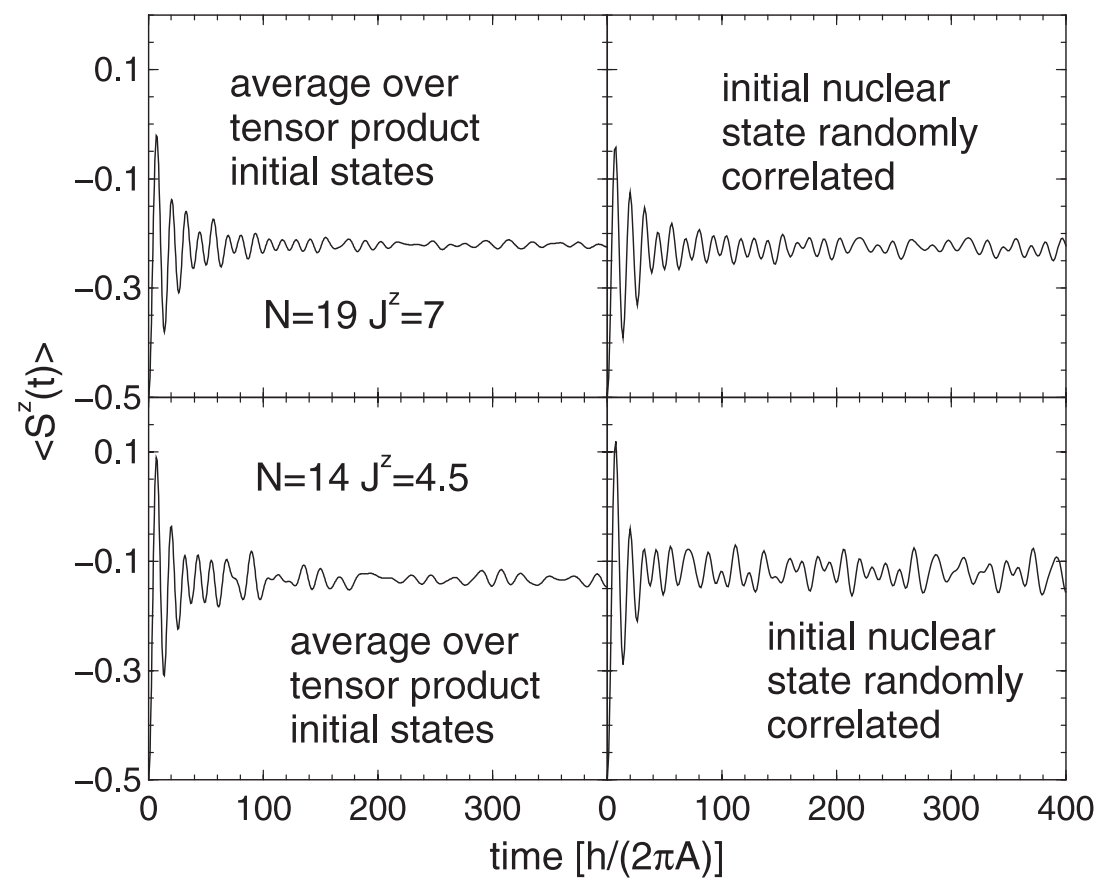

Figure 8. The two left panels show the electron spin dynamics averaged over all possible initial tensor product states for two different system sizes and degrees of polarization. The two right panels show the corresponding data for a single randomly correlated initial condition for the nuclear system. As the comparison shows, the time evolution for the randomly correlated nuclear spin system closely mimics the average over all tensor product initial states.

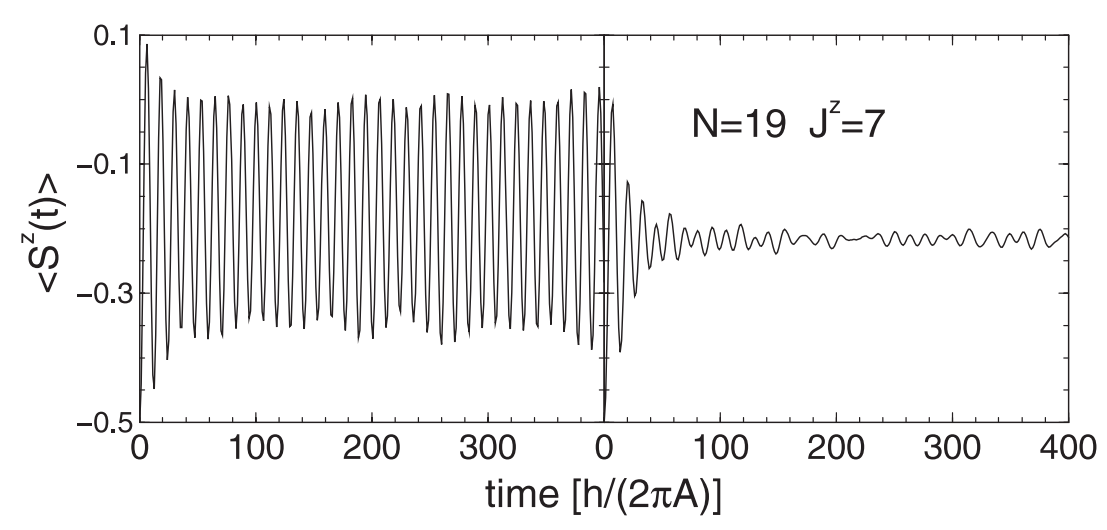

Figure 9. Time evolution of $\left\langle S^{z}(t)\right\rangle$ for two types of initially randomly correlated nuclear spin states. In the left panel the amplitudes $\alpha_{T}$ are restricted to have non-negative real and imaginary parts, while in the right panel they all have the same modulus but completely random phases.

However, these are tiny in magnitude and oscillate around zero. For an initial tensor product state these transverse components can become sizable and the time evolution again strongly depends on the concrete initial tensor product state. Moreover, as mentioned earlier, the exact form of the coupling constants is also not crucial as long as they are sufficiently inhomogeneous. For instance, we obtain qualitatively the same results if we choose the coupling parameters randomly from a uniform distribution. 


\subsection{Decoherence and the generation of entanglement}

In circumstances of quantum information processing the decay of a qubit is usually viewed as some 'decoherence' process due to the environment attacking the quantum information. As seen above, the spin decay is generically slower if the spin environment is initially in a uncorrelated state. This finding suggests that it is advantageous for protecting quantum information to disentangle the environment that unavoidably interacts with the qubit system.

A 'decoherence' process of the above kind can be viewed as the generation of entanglement between a qubit and its environment. The system investigated here provides an illustrative example for this statement. The entanglement in the total state $|\psi(t)\rangle$ between the central electron spin and its environment can be measured by the von Neumann entropy of the partial density matrix where either the electron or the environment has been traced out from the pure-state density matrix $|\psi(t)\rangle\langle\psi(t)|[65]$. Tracing out the nuclear system we have

$$
\rho_{\mathrm{el}}(t)=\left(\begin{array}{cc}
\frac{1}{2}+\left\langle S^{z}(t)\right\rangle & \left\langle S^{+}(t)\right\rangle \\
\left\langle S^{-}(t)\right\rangle & \frac{1}{2}-\left\langle S^{z}(t)\right\rangle
\end{array}\right) .
$$

This matrix has eigenvalues $\lambda_{ \pm}=1 / 2 \pm|\langle\vec{S}(t)\rangle|$ and the measure of entanglement is $E(|\psi(t)\rangle)=-\lambda_{+} \log \lambda_{+}-\lambda_{-} \log \lambda_{-}$. Thus, the formation of expectation values $|\langle\vec{S}(t)\rangle| \neq 1 / 2$ (or, in the case of fixed $J^{z}$, just $\left|\left\langle S^{z}(t)\right\rangle\right| \neq 1 / 2$ ) is a manifestation of the entanglement between the electron spin and the nuclear spin system. The maximum entanglement, $E=\log 2$, is achieved if the electron spin has decayed completely, as measured by the expectation values of its components, $\langle\vec{S}(t)\rangle=0$. The generation of quantum entanglement between the electron spin and the nuclear spin system signalled by a reduced value of $\langle\vec{S}(t)\rangle$ is a main crucial difference between the quantum system studied here and its classical 'counterpart' described by a system of Landau-Lifshitz equations. These equations can be obtained from the Heisenberg equations of motion for the quantum system, $\partial \vec{S} / \partial t=i[\mathcal{H}, \vec{S}] / \hbar, \partial \vec{I}_{i} / \partial t=i\left[\mathcal{H}, \vec{I}_{i}\right] / \hbar$, by performing expectation values on both sides within spin-coherent states and assuming that the expectation values of all operator products factorize to products of expectation values. This procedure becomes exact in the classical limit [66]. The resulting equations do not contain operators any more but just describe the dynamics of three-component vectors (classical spins) of fixed length. We have performed simulations of such a classical spin system by solving the Landau-Lifshitz equation via the fourth-order Runge-Kutta scheme. As a result, the central classical spin performs an irregular chaotic motion which does not show any similarity to the results for the quantum spin-1/2 case. In particular all qualitative features of quantum effects such as the generation of entanglement (signalled by a decay of spins as measured by their expectation values) are not present in such a time evolution. Therefore the Landau-Lifshitz equation provides only a rather poor description of the underlying quantum system.

We finally consider the nuclear spin correlator $C(t)=\left\langle I^{z}(t) I^{z}(0)\right\rangle, \vec{I}=\sum_{i} \vec{I}_{i}$, which can be measured directly by local NMR, like measurements such as magnetic resonance force microscopy [67]. In a subspace of given $J^{z}$ and the electron spin pointing downwards initially this quantity is $C(t)=\left(J^{z}-\left\langle S^{z}(t)\right\rangle\right)\left(J^{z}+1 / 2\right)$. A realistic initial state will have its dominant weight in a series of subspaces with neighbouring $J^{z}$ centred around some value. Then the time evolution of $\left\langle S^{z}(t)\right\rangle$ is very similar in these subspaces and the dynamics of the total nuclear spin can be mapped out by measuring the electron spin and vice versa.

\subsection{Averaging over nuclear configurations. Dephasing time for an ensemble of dots}

In section 4.1.1 we have seen (see also section 4.6.1) that the decay of $\left\langle S^{z}(t)\right\rangle$ for the initial tensor product state occurs starting from the time $t>\hbar N / A$, with $\hbar N / A \simeq 10^{-6} \mathrm{~s}$ in GaAs dots. On the other hand, the electron spin precesses in the net nuclear field with the 
characteristic period $\omega_{\mathrm{N}}^{-1} \simeq \hbar \sqrt{N} / A \simeq 10^{-8}-10^{-9} \mathrm{~s}$. Thus we see that the electron spin undergoes many precessions in a given nuclear field $\vec{h}_{\mathrm{N}}=\sum_{i} A_{i}\left\langle\vec{I}_{i}\right\rangle$ (for a given nuclear configuration) before decoherence sets in due to the non-uniform hyperfine couplings $A_{i}$. This behaviour changes dramatically when we average over nuclear configurations [24]. Let us average $C_{T}(t)=\left\langle T\left|S^{z}(t)-S^{z}(0)\right| T\right\rangle$ over all initial tensor product nuclear configurations $|T\rangle$. For that purpose we calculate $C_{T}(t)$ exactly by treating the nuclear field purely classically, i.e. as a c-number. Then we obtain

$$
C_{T}(t)=\frac{h_{\mathrm{N}_{\perp}}^{2}}{2 h_{\mathrm{N}}^{2}}\left(1-\cos \left(h_{\mathrm{N}} t\right)\right),
$$

where $h_{\mathrm{N}}=\sqrt{h_{\mathrm{N}_{z}}^{2}+h_{\mathrm{N}_{\perp}}^{2}}$ is the nuclear field, with $h_{\mathrm{N}_{\perp}}^{2}=h_{\mathrm{N}_{x}}^{2}+h_{\mathrm{N}_{y}}^{2}$. Again, the value of $h_{\mathrm{N}}$ corresponds to a given nuclear tensor product state $|T\rangle$. We average equation (29) over a Gaussian distribution for $h_{\mathrm{N}}$, i.e. over $P\left(h_{\mathrm{N}}\right) \propto \exp \left(-3 h_{\mathrm{N}}^{2} / 2 \omega_{\mathrm{N}}^{2}\right)$. Defining $C_{\mathrm{cl}}(t)=$ $\int \mathrm{d} h_{\mathrm{N}} P\left(h_{\mathrm{N}}\right) C_{T}(t)$, we obtain

$$
C_{\mathrm{cl}}(t)=\frac{1}{3}\left[1+\left(\frac{\omega_{\mathrm{N}}^{2} t^{2}}{3}-1\right) \mathrm{e}^{-\omega_{\mathrm{N}}^{2} t^{2} / 6}\right] .
$$

Thus we get a very rapid (Gaussian) decay of $C_{\mathrm{cl}}(t)$ for $t \gg \omega_{\mathrm{N}}^{-1}$, which means that the dephasing time is $\sqrt{N} / A$. From the above equation we see that $\left\langle S^{z}(t)\right\rangle$ saturates at $1 / 3$ of its initial value of $-1 / 2$. An important approximation used here is the classical treatment of the nuclear field as a c-number (not an operator). The important qualitative point we want to illustrate here is the difference between the decoherence of an electron in an individual quantum dot compared to the case of an ensemble of dots.

Investigations similar in spirit to the above considerations were performed by Merkulov et al [30] who have also considered the problem of the electron spin dephasing due to the hyperfine interaction for an ensemble of dots. As was already mentioned above, the field exerted on the electron spin by hyperfine interaction with surrounding nuclei is typically much larger at $N \gg 1$ than the field the electron provides to an individual nucleus. Following this observation Merkulov et al [30] observed several timescales with different decay laws. At times shorter than $\hbar N / A$ they have considered an approximation where the nuclear spins are assumed to be static on the typical timescale of the electron spin dynamics. That is, in an individual quantum dot the electron spin dynamics is approximated by a coherent rotation in the hyperfine field provided by the 'frozen' nuclear spin configuration. Dephasing of the electron spin is then obtained by averaging over an ensemble of dots with individual nuclear spin configurations. Then they obtained essentially the same formula, see equation (30), with the same timescale- $\hbar \sqrt{N} / A$. The resulting dephasing times $T_{2}^{*}$ obtained in [30] are in reasonable agreement with experiments $[14,15]$.

Note that, at a later timescale $(t \gg \hbar N / A)$, the authors of [30] obtained further slow electron spin decay by considering the variations of the nuclear field direction when the magnitude of the field is conserved, which is correct at $N \gg 1$.

\subsection{Further approximate studies of electron spin dynamics}

The exact solution for the electron spin dynamics under the hyperfine coupling (3) obtained in [24] and described briefly in section 4.1.1 is unfortunately restricted to the case of an initially fully polarized nuclear spin system. The numerical approach described in section 4.3 allows for a (quasi-)approximation-free treatment of systems with an arbitrary degree of initial nuclear polarization but is, in particular at lower polarization, restricted to rather small systems. Therefore, in order to investigate the physically most interesting case of larger systems with 
initially moderate or even very low nuclear polarization, one needs to resort to approximations. In this section we review several recent approaches.

4.6.1. Perturbation theory. The contribution to the hyperfine Hamiltonian (3) coupling to the $z$ component of the electron spin:

$$
\mathcal{H}_{0}=g \mu_{\mathrm{B}} S^{z} B+S^{z} \sum_{i} A_{i} I_{i}^{z}
$$

is diagonal in a basis of tensor product states with respect to the $z$ axis as formulated in equation (22). The remaining part of the Hamiltonian:

$$
V=\sum_{i} \frac{A_{i}}{2}\left(S^{+} I_{i}^{-}+S^{-} I_{i}^{+}\right),
$$

was treated in $[24,25]$ as a perturbation to $\mathcal{H}_{0}$. Again we shall concentrate on the case of nuclear spins of length $1 / 2$. Assuming the system to be initially $(t=0)$ in a tensor product state with respect to the $z$ axis ( $\mathrm{cf}(22)$ ) with the electron spin, as before, pointing downwards, the lowest nonvanishing contribution to the electron spin dynamics in time-dependent perturbation theory is of second order in $V$. Specifically, one finds

$$
\left\langle S^{z}(t)\right\rangle=-\frac{1}{2}+2 \sum_{k} \frac{\left|V_{i k}\right|^{2}}{\omega_{i k}^{2}}\left(1-\cos \left(\omega_{i k} t\right)\right),
$$

where the summation goes over all intermediate states $|k\rangle$ which are tensor product states of the form (22). $V_{i k}$ is the matrix element of $V$ between an intermediate state $\mid k=\Uparrow,\left\{\ldots, I_{z}^{k}=\right.$ $-1 / 2, \ldots\}\rangle$ and the initial state $\left|i=\Downarrow,\left\{\ldots, I_{z}^{k}=+1 / 2, \ldots\right\}\right\rangle$ and $\omega_{i k}=\left(\varepsilon_{i}-\varepsilon_{k}\right) / \hbar$, where $\varepsilon_{i}$ and $\varepsilon_{k}$ are the eigenvalues of $|i\rangle$ and $|k\rangle$, respectively, with respect to $\mathcal{H}_{0}$.

Evaluating this lowest-order contribution for a large $(N \gg 1)$ unpolarized system, one finds a universal power law for the decay of $\left\langle S^{z}(t)\right\rangle$ : for times large compared to $\hbar N / A$, $\left|\left\langle S^{z}(t)\right\rangle\right|$ decays as $t^{-3 / 2}$. This is a central finding within this perturbative approach and agrees with the perturbative limit (i.e. large Zeeman field) of the exact solution in the fully polarized case discussed in section 4.1.1. Note that for a weak Zeeman field $\epsilon_{z}<A / \sqrt{N}$ the part of the electron spin state which decays is of the order of the initial value, in contrast to the fully polarized case where this part is of the order of $1 / N$, see figure 2 .

The perturbative approach has the following shortcomings: clearly, $\mathcal{H}_{0}$ and $V$ are of the same order of magnitude if one does not apply a very large external magnetic field. Therefore, there is, in general, no small parameter justifying a perturbative expansion concentrating on low orders and one would need to sum over higher orders, provided that this perturbative series has sufficient convergence properties. In higher orders, however, one encounters increasing divergences due to vanishing denominators in the perturbative contributions [24]. Therefore, the findings obtained from the low-order result (33) might appear not very reliable. Indeed, from the exact solution in the fully polarized case [24,25] (cf section 4.1.1) one finds a different (not a power) law for spin decay in the limit of low external magnetic field. However, there is a reasonable hope that the basic conclusion from the low-order perturbative approach is still correct, namely that a non-uniform hyperfine coupling leads to a non-exponential spin decay.

Moreover, the perturbative approach can, for technical reasons, only deal with initial states where the nuclear spin system is in a tensor product state. As seen in section 4.3 the behaviour of such a type of initial state depends significantly on the particular initial condition, in contrast to the behaviour of randomly correlated initial states. 
4.6.2. Studies using Markovian approximations to the nuclear spin dynamics. Saykin et al [31] and de Sousa and Das Sarma [28, 29] have performed investigations using, among other simplifying assumptions, Markovian approximations to describe the dynamics of the nuclear spins.

In [31] the situation of an electron bound to ${ }^{31} \mathrm{P}$ donor in a silicon matrix was studied. The electron spin interacts with the central ${ }^{31} \mathrm{P}$ and the surrounding ${ }^{29} \mathrm{Si}$ nuclear spins. Then the authors formulate a master equation for the reduced electron density matrix where the nuclear spin dynamics is governed by a Markov process. Moreover, the total density matrix is assumed to be separable at all times with respect to the subsystems given by the electron spin and the nuclear spin bath and the density operator of the latter system is assumed to be timeindependent. Due to the first assumption, decoherence due to the formation of entanglement, as described in section 4.4 , is excluded since the total density matrix is taken to be always separable [64]. As a result, the authors find an exponential decay of the elements of the reduced density matrix to their equilibrium values, where the longitudinal relaxation time $T_{1}$ and the transversal dephasing time $T_{2}$ (with respect to the direction of a weak external field) fulfil the relation $T_{1}=T_{2} / 2$. However, as already explained in section 4.1.1, the application of the Markov approximation in this situation is not justified, which might explain that this result differs qualitatively from the findings from the exact solution in the case of an initially fully polarized nuclear spin system (cf section 4.1.1) and the perturbative result for the unpolarized case (cf section 4.6.1), where a power law decay was found.

In $[28,29]$ the Hamiltonian (3) was studied under the assumption of strong external magnetic field coupled to the electron spin and suppressing the spin-flip terms. Therefore, the hyperfine interaction is approximated by (31). This approach is applicable at an external Zeeman magnetic field which is much larger than the internal nuclear field, i.e. $\varepsilon_{z} \gg$ $\hbar \omega_{\mathrm{N}} \simeq A / \sqrt{N}$. We recall that the part of the initial electron spin state which decays due to inhomogeneous hyperfine coupling is small under the above conditions: this part is of the order of $\left(\hbar \omega_{\mathrm{N}} / \varepsilon_{z}\right)^{2} \ll 1$, see [24, 25]. The authors of [28, 29] then introduced the nontrivial dynamics through the dipolar interaction among the nuclear spins. This interaction is taken into account in a truncated version, neglecting terms that change the total spin component in the direction of the external field, consistent with the earlier approximation. Thus, in this approximation the dipolar dynamics among the nuclear spins induces the decay of the electron spin. As a further assumption, the nuclear spin system is then approximated as a fluctuating field generated by a Markov process. After performing a detailed mathematical analysis of this resulting effective model [29] the authors conclude that the electron spin decay will occur on essentially the same timescale as the dipolar interaction among the nuclear spins, i.e. $T_{n 2} \simeq 10^{-4}-10^{-5} \mathrm{~s}$. Given the various assumptions leading to this effective model, this is a very natural result.

Applying their findings to the situation of an electron bound to a ${ }^{31} \mathrm{P}$ donor embedded in an Si crystal, the authors find an interesting dependence of the spin memory time $T_{\mathrm{M}}$ (as measured by spin-echo experiments) on the direction of the external field relative to the crystal. This effect is induced by the directional dependence of the dipolar interaction and provides a possibility to experimentally probe these results and the underlying assumptions [44].

\subsection{Further studies and developments}

We now summarize further, mostly theoretical, work relevant to the issue of hyperfine interaction between an electron spin bound to a quantum dot and surrounding nuclear spins.

The relaxation rate of the longitudinal electron spin component, i.e. $1 / T_{1}$, which is due to the interplay between hyperfine interaction and dissipative phonon processes was studied by 
Erlingsson et al [23] and Erlingsson and Nazarov [27]. The approach in [27] is a semiclassical one, introducing an internal field due to the nuclear spins which acts on the electron spin in addition to an external magnetic field. The observed relaxation rate is very small, though this mechanism can prevail over the mechanism which is the interplay of the spin-orbit interaction and the spin-independent interaction with phonons [20]. It happens at very low external magnetic fields when the corresponding $T_{1}$ time is of the order of $100 \mathrm{~s}$. The analogous problem of the calculation of the $T_{2}$ time which is due to the combined effect of hyperfine interaction and phonon processes was considered recently by Semenov and Kim [40]. These authors noticed that, due to thermal fluctuations, the electron can make spin-conserving transitions between different orbital states. Then, because of either the energy dependence of the $g$ factor or different nuclear fields seen by the electron spin in different orbital states, these fluctuations lead to electron spin decoherence (since the precession frequencies are different in different states). These mechanisms are only important at relatively high temperatures since they are exponentially suppressed at temperatures much smaller than the energy distance between the neighbouring orbital states. For example, in the case of the combined effect of hyperfine interaction and phonon processes for typical GaAs quantum dots the decoherence time is of the order of $10 \mathrm{~s}$ at temperature $1 \mathrm{~K}$.

Lyanda-Geller et al [26] have investigated the dependence of the relaxation rate of nuclear spins in a quantum dot on the electronic state of the dot and concluded that the effects of a Coulomb blockade should also be observable in nuclear relaxation in such systems.

A proposal for using the nuclear spin system as a long-lived memory for information originally contained in the electron spin qubit was put forward by Taylor et al [37]. In a related study, Imamoglu et al [38] have proposed an all-optical scheme for polarizing the nuclear spins by manipulating the electron spin.

Saikin and Fedichkin [35] have investigated the influence of hyperfine interactions on gate operations within the Si:P quantum computing proposal due to Kane [5].

The possibility of nuclear spins forming an effective quantum dot, confining electrons through hyperfine interaction, was proposed recently by Pershin [39].

Theoretical studies on the decoherence of a two-level system coupled to surrounding spins were also presented recently by Frasca [68]. These studies stem from a somewhat different context but are similar in spirit to the ones reported on in this review. We also mention a recent numerical study by Dobrovitski et al [69] on spin dynamics, stressing the role of entropy. There a central spin is coupled inhomogeneously to an essentially non-interacting spin environment where an Ising-like coupling was used. To allow for nontrivial dynamics the authors introduced a magnetic field perpendicular to the $z$ direction of the Ising coupling. In a very recent paper [70] the same authors have numerically studied the damping of quantum oscillations in the system of two central spins. These central spins are coupled by strong Heisenberg exchange. This system in turn is coupled to a spin bath (which can be a nuclear system) through inhomogeneous Heisenberg interaction, a scenario similar to the one studied in [3]. The state of the bath is initially a random superposition of all possible basis states. Assuming the coupling constants $A_{k}$ are random, in the system of $N=13$ bath spins the authors observed a two-step decoherence process. Initially, decoherence is very fast and after the first step the oscillations of $z$ components of the central spins $S_{1}^{z}(t)$ and $S_{2}^{z}(t)$ persist and decay very slowly. It is interesting that the authors have managed to fit the first step's fast decay by equation (30), see section 4.5 , which probably confirms the self-averaging occurring for randomly correlated initial nuclear states described in section 4.3.3. The second slow step of the decoherence process is presumably the analogue of the decay described in sections 4.1.1 and 4.6.1. 


\section{Conclusions and outlook}

We have reviewed the recent literature on electron spin dynamics in semiconductor nanostructures due to hyperfine interaction with surrounding nuclear spins. This issue is of particular interest with respect to several proposals for quantum information processing in solid state systems. Although the basic Hamiltonian (3) looks rather simple it describes an intricate many-body problem which, in general, does not seem to allow for an analytical solution.

In the case of an initially fully polarized nuclear spin system an exact analytical solution for the spin dynamics can be found. For not completely polarized nuclei approximationfree results can only be obtained numerically in small model systems. We have compared these exact results with findings from several approximation strategies such as perturbation theory and (semi-)classical approximations to the nuclear spin dynamics, including Markovian approximations.

Many of those approximations are not particularly well controlled and they suppress important features of the full quantum system. For instance, the pronounced dependence of the dynamics of the electron spin on the type of initial conditions for the nuclear system is not reproduced by any of the approximation strategies which have appeared so far. Therefore, the most obvious direction for future work is the development of possibly systematically controlled approximation techniques which reproduce important features of the full quantum dynamics and allow reliable predictions for realistic systems. A possible, but presumably technically quite complicated, route for such future work is the Bethe ansatz solution outlined in section 4.1.2. Such progress towards more reliable predictions for experimentally relevant situations is especially desirable because of the importance of this issue to several proposals for quantum information processing in a semiconductor environment, see in particular [3, 5], and possibly also for other scenarios in the emerging field of spin electronics $[1,2]$.

\section{Acknowledgments}

We thank B L Altshuler, W A Coish, S I Erlingsson, Al L Efros L Glazman, F Göhmann, V N Golovach, Y N Nazarov and D Saraga for collaborations and/or discussions. This work was supported by NCCR Nanoscience, the Swiss NSF, DARPA and ARO.

\section{References}

[1] Wolf S A, Awschalom D D, Buhrman R A, Daughton J M, von Molnar S, Roukes M L, Chtchelkanova A Y and Treger D M 2001 Science 2941488

[2] Awschalom D D, Loss D and Samarth N (ed) 2002 Semiconductor Spintronics and Quantum Computation (Berlin: Springer)

[3] Loss D and DiVincenzo D P 1998 Phys. Rev. A 57120

[4] Privman V, Vagner I D and Kventsel G 1998 Phys. Lett. A 239141

[5] Kane B E 1998 Nature 393133

[6] Barnes C H W, Shilton J M and Robinson A M 2000 Phys. Rev. B 628410

[7] Levy J 2001 Phys. Rev. A 64052306

[8] Ladd T D, Goldman J R, Yamaguchi F, Yamamoto Y, Abe E and Itoh K M 2002 Phys. Rev. Lett. 89017901

[9] Haller E E 1995 J. Appl. Phys. 772857

[10] Kikkawa J M and Awschalom D D 1998 Phys. Rev. Lett. 804313

[11] Kikkawa J M and Awschalom D D 1999 Nature 397139

[12] Gammon D, Snow E S, Shannabrook B V, Katzer D S and Park D 1996 Phys. Rev. Lett. 763005

[13] Gammon D, Efros A L, Kennedy T A, Rosen M, Katzer D S, Park D, Broen S W, Korenev V L and Merkulov I A 2001 Phys. Rev. Lett. 865176 
[14] Gupta J A, Awschalom D D, Peng Y and Alivisatos A P 1999 Phys. Rev. B 59 R10421

[15] Epstein R J, Fuchs D T, Schoenfeld W V, Petroff P M and Awschalom D D 2001 Appl. Phys. Lett. 78733

[16] Fujisawa T, Tokura Y and Hirayama Y 2001 Phys. Rev. B 63 R081304 Fujisawa T, Austing D G, Tokura Y, Hirayama Y and Tarucha S 2002 Phys. Rev. Lett. 88236802

[17] Hanson R, Witkamp B, Vandersypen L M K, Willems van Beveren L H, Elzerman J M and Kouwenhoven L P 2003 Phys. Rev. Lett. 91196802

[18] Burkard G, Loss D and DiVincenzo D P 1999 Phys. Rev. B 592070

[19] Khaetskii A V and Nazarov Y V 2000 Phys. Rev. B 6112639

[20] Khaetskii A V and Nazarov Y V 2001 Phys. Rev. B 64125316 Khaetskii A 2001 Physica E 1027

[21] Halperin B I, Stern A, Oreg Y, Cremers J N H J, Folk J A and Marcus C M 2001 Phys. Rev. Lett. 862106

[22] Khaetskii A, Loss D and Glazman L 2003 J. Supercond. 16221

[23] Erlingsson S I, Nazarov Y V and Falko V I 2001 Phys. Rev. B 64195306

[24] Khaetskii A V, Loss D and Glazman L 2002 Phys. Rev. Lett. 88186802

[25] Khaetskii A V, Loss D and Glazman L 2003 Phys. Rev. B 67195329

[26] Lyanda-Geller Y B, Aleiner I L and Altshuler B L 2002 Phys. Rev. Lett. 89107602

[27] Erlingsson S I and Nazarov Y V 2002 Phys. Rev. B 66155327

[28] de Sousa R and Das Sarma S 2003 Phys. Rev. B 67033301

[29] de Sousa R and Das Sarma S 2002 Preprint cond-mat/0211567

[30] Merkulov I A, Efros A L and Rosen M 2002 Phys. Rev. B 65205309

[31] Saykin S, Mozyrsky D and Privman V 2002 Nano Lett. 2651

[32] Schliemann J, Khaetskii A V and Loss D 2002 Phys. Rev. B 66245303

[33] Eto M 2002 Preprint cond-mat/0210231

[34] Semenov Y G and Kim K W 2003 Phys. Rev. B 67073301

[35] Saikin S and Fedichkin L 2003 Phys. Rev. B 67161302

[36] Pershin Y V and Privman V 2003 Nano Lett. 3695

[37] Taylor J M, Marcus C M and Lukin M D 2003 Phys. Rev. Lett. 90206803

[38] Imamoglu A, Knill E, Tian L and Zoller P 2003 Phys. Rev. Lett. 91017402

[39] Pershin Y V 2003 Preprint cond-mat/0305002

[40] Semenov Y G and Kim K W 2003 Preprint cond-mat/0305005

[41] Feher G and Gere E A 1959 Phys. Rev. 1141245

[42] Gordon J P and Bowers K D 1958 Phys. Rev. Lett. 1368

[43] Chiba M and Hirai A 1972 J. Phys. Soc. Japan 33730

[44] Tyryshkin A M, Lyon S A, Astashkin A V and Raitsimring A M 2003 Preprint cond-mat/0303006

[45] Salis G, Fuchs D T, Kikkawa J M, Awschalom D D, Ohno Y and Ohno H 2001 Phys. Rev. Lett. 862677

[46] Eickhoff M, Lenzman B, Flinn G and Suter D 2002 Phys. Rev. B 65125301 Eickhoff M, Lenzmann B, Suter D, Hayes S E and Wieck A D 2003 Phys. Rev. B 67085308

[47] Fermi E 1930 Z. Phys. 60320

[48] Pikus G E and Titkov A N 1984 Spin relaxation under optical orientation in semiconductors Optical Orientation ed F Meier and B P Zakharchenya (Amsterdam: Elsevier) p 73

[49] Abragam A 1961 The Principles of Nuclear Magnetism (Oxford: Clarendon)

[50] Slichter C P 1990 Principles of Magnetic Resonance (Berlin: Springer)

[51] Gueron M 1964 Phys. Rev. A 135200

[52] Paget D, Lampel G, Sapoval B and Safarov V I 1977 Phys. Rev. B 155780

[53] Shulman R G and Wyluda B J 1956 Phys. Rev. 1031127

[54] Gaudin M 1976 J. Phys. (Paris) 371087

[55] Garajeu D 2002 J. Math. Phys. 435732

[56] Sklyanin E K and Takebe T 1996 Phys. Lett. A 219217

[57] Richardson R W and Sherman N 1964 Nucl. Phys. 5222

[58] Amico L, Di Lorenzo A and Osterloh A 2001 Phys. Rev. Lett. 865759

[59] Schechter M, Imry Y, Levinson Y and von Delft J 2001 Phys. Rev. B 63214518

[60] von Delft J and Poghossian R 2002 Phys. Rev. B 66134502

[61] Yuzbashyan E A, Baytin A A and Altshuler B L 2003 Preprint cond-mat/0305635

[62] Sklyanin E K 1999 Lett. Math. Phys. 47275

[63] Schrödinger E 1935 Naturwissenschaften 23807

[64] For a general overview on quantum information theory see, for example, Nielsen M A and Chuang I L 2000 Quantum Computation and Quantum Information (Cambridge: Cambridge University Press)

[65] Bennett C H, Bernstein H J, Popescu S and Schumacher B 1996 Phys. Rev. A 532046 
[66] Schliemann J and Mertens F G 1998 J. Phys.: Condens. Matter 101091

[67] For a recent overview see Suter A, Pelekhov D V, Roukes M L and Hammel P C 2002 J. Magn. Reson. 154 210 and references therein

[68] Frasca M 2002 Physica E 15252

[69] Dobrovitski V V, De Raedt H A, Katsnelson M I and Harmon B N 2001 Preprint quant-ph/0112053

[70] Dobrovitski V V, De Raedt H A, Katsnelson M I and Harmon B N 2003 Phys. Rev. Lett. 90210401

[71] American Institute of Physics Handbook 1972 3rd edn (New York: McGraw-Hill) 